\title{
Design of Command Shaper using Gain-delay Units and Particle Swarm Optimisation Algorithm for Vibration Control of Flexible Systems
}

\author{
M. S. Alam \\ Department of Applied Physics, Electronics and Communication Engineering, The University of Dhaka, Bangladesh
}

M. O. Tokhi ${ }^{\dagger}$
Department of Automatic Control and Systems Engineering, The University of Sheffield, UK

(Received 19 November 2006; accepted 29 June 2007)

\begin{abstract}
Conventional command shaping methods involve convolving a desired command with a sequence of impulses that may prove computationally expensive or unsuitable for online applications. Moreover, a priori knowledge of the system parameters, such as resonance frequencies and associated damping ratios, is required to design the exact sequence of impulses to produce a command that results in zero residual vibration. A new command shaping method is proposed in this paper using gain and delay units. Assuming that, no prior information is available about the system, a new variant of particle swarm optimisation (PSO) algorithm is proposed and used to derive the gain and delay values. The effect of the total number of delay and gain units is also analysed. Moreover, an adaptive control strategy is developed based on the proposed command shaping technique where the PSO is used to adjust the controller parameters online. A twin rotor (experimental flexible) system is used for assessment and evaluation of the control strategy. The effectiveness of the technique is assessed both in the time domain and the frequency domains.
\end{abstract}

${ }^{\dagger}$ Member of the International Institute of Acoustics and Vibration (IIAV)

\section{INTRODUCTION}

Flexible systems are lighter, faster and less expensive than rigid ones, but they pose various challenges as compared to their rigid counterparts. These include system design, vibration control and structural optimisation. The advantages of such systems are often marred by the structural vibration originating, mainly, from system flexibility while in operation. In order to achieve high-speed and accurate positioning, it is necessary to control the system's vibratory response in a cost-effective manner. Numerous techniques have been proposed to effectively control flexible systems. These can be divided into two broad categories, namely feedback control and feedforward control. A good literature review can be found in reference. ${ }^{1}$

A number of feedforward control approaches have been proposed for flexible systems. Command shaping based on various filtering techniques such as low-pass, band-stop and notch filters have been reported and proved to be effective in practical systems. ${ }^{2}$ In this approach, the input torque is modified or shaped so that it contains as little as possible energy at and around the natural frequencies of the system. A feedforward control scheme based on the input command shaping, introduced by Singer and Seering, ${ }^{3}$ has been applied to the control of different types of flexible systems for vibration reduction or trajectory tracking or occasionally both.,

Singh and Vadali ${ }^{6}$ presented a method to minimise residual vibration of structures or lightly damped servomechanisms using multiple time delays in conjunction with a proportional part. In order to increase robustness of the control- ler, they included a basic single time-delay control unit in cascade. In another work, the authors presented a design procedure of open-loop controllers to reduce residual vibration in flexible structures using a multiple step inputs delayed in time. ${ }^{7}$ The controller attenuated the residual vibration by cancelling the complex poles of the system and robustness was achieved by locating additional zeros at the cancelled poles of the system. The paper also investigated a design procedure of robust time-delay controllers for multiple modes with user selected time delays. Moreover, a design method for the minimum time-delay controller was also proposed where step input magnitude values were constrained within 0 and $1 .^{7,8} \mathrm{~A}$ single-link flexible arm robot was used to illustrate the effectiveness of the controller.

Bodson' presented an algorithm for the tuning of two input shaping methods to prevent the excitation of oscillatory modes in resonant systems. Efforts have been made to make a command shaper to adapt to unknown system parameters using adaptive filters, ${ }^{10}$ but conventional adaptive algorithms have the drawback of local minima which may result in poor vibration reduction. Alam et al. ${ }^{11}$ investigated the potential of genetic algorithms $(\mathrm{GAs})^{12}$ in designing uni-modal command shapers for a twin rotor system that resulted in a significant reduction in vibration at the cost of introducing a long response delay. The command shaping technique, in practice, causes delay in the system's response while it reduces vibration and the amount of reduction in vibration and rise time are often in conflict with one another. The weighted sum of these two competing objectives was selected heuristically and used to reduce system vibration as well as to obtain a satisfactory response rise-time. ${ }^{13}$ The success of the approach 